\title{
The optics and physics of near infrared imaging
}

\section{Michael Vollmer, Klaus-Peter Möllmann, Joseph Shaw}

Michael Vollmer, Klaus-Peter Möllmann, Joseph A. Shaw, "The optics and physics of near infrared imaging," Proc. SPIE 9793, Education and Training in Optics and Photonics: ETOP 2015, $97930 Z$ (8 October 2015); doi: $10.1117 / 12.2223094$

SPIE Event: Education and Training in Optics and Photonics: ETOP 2015, 2015, Bordeaux, France 


\title{
The optics and physics of near infrared imaging
}

\author{
Michael Vollmer ${ }^{\mathrm{a}}$, Klaus-Peter Möllmann ${ }^{\mathrm{a}}$, Joseph A. Shaw ${ }^{\mathrm{b}}$ \\ ${ }^{a}$ Microsys. and Opt. Techn., Univ. Appl. Sci. Brandenburg, 14770 Brandenburg/Germany; \\ bElectrical and Computer Engineering, Montana State University, Bozeman, Montana 59717, USA
}

\begin{abstract}
A large part of photonics research and development, as well as commercial applications such as optical data transmission or infrared thermal imaging, occurs in the infrared spectral range between $0.8 \mu \mathrm{m}$ and $15 \mu \mathrm{m}$. However, relatively little material is so far available for experimentally teaching the physics and optics of this spectral range. We report a respective new approach in the near infrared (NIR) range between $0.8 \mu \mathrm{m}$ and $1.7 \mu \mathrm{m}$ that allows visualization of a number of fascinating physics phenomena. First, we use the near-infrared sensitivity of silicon-based detectors in rather inexpensive video cameras and digital single-lens reflex cameras by removing the infrared-blocking filter and replacing it with a visible-radiation blocking filter. Second, we utilize modern NIR cameras based on InGaAs detectors. With both camera types we illustrate and explain a number of physics concepts that are especially suitable for curricula in optics and photonics. Examples include the strangely bright appearance of vegetation, contrast enhancement between clouds and sky, the initially surprising differences of optical material properties between the VIS and NIR range, the possibilities of visualizing buried hidden structures and texts, and recent medical applications to locate blood vessels below the skin.
\end{abstract}

Keywords: optics education, infrared photography, near infrared imaging, NIR radiation, detection, and material properties

\section{1) INTRODUCTION}

Human perception is limited to the visible spectral range (VIS) that is defined by the luminous efficiency functions to range between wavelengths of $\lambda=380 \mathrm{~nm}$ and $\lambda=780 \mathrm{~nm}$. In contrast, Si-based sensors in cameras and camcorders have sensitivities extending to about $1100 \mathrm{~nm}$, and more expensive specialized commercial detector systems in IR cameras are available for the thermal infrared wavelengths up to about $15 \mu \mathrm{m}$ [1]. In optics teaching, the classical content refers primarily to the VIS spectral range, while special courses deal with infrared technologies, usually extending from around $1.5 \mu \mathrm{m}$ up to $15 \mu \mathrm{m}$ for imaging systems (e.g. [2]) and up to about $25 \mu \mathrm{m}$ for spectroscopy (e.g. [3]). The intermediate near infrared range (NIR) between 0.8 and $1.7 \mu \mathrm{m}$ has long been neglected in education, probably due to the lack of suitable inexpensive instruments and experiments illustrating the physical effects at these wavelengths.

Recently it has, however, been demonstrated [4,5] that rather simple experiments are available for teaching the fascinating physics phenomena occurring at NIR wavelengths. Most simply, one may use regular compact digital cameras or DSLR cameras with Si-based detector chips. In order to match the appearance of photos to the human visual sensation, the NIR part of the radiation incident on cameras is usually blocked by an IR cut-off filter. If this filter is removed, the incident radiation also contains the NIR radiation. If an additional filter to block the VIS light is used, the modified camera then only detects NIR radiation between, e.g., $800 \mathrm{~nm}$ and $1100 \mathrm{~nm}$, which can lead to strange effects in the appearance of photos of wellknown objects. This realm of NIR photography is also exploited by amateur photographers [6].

It is quite easy to record NIR photos; however, it is a different task to understand why certain objects appear different in NIR light compared to the VIS range. Here we try to fill part of the gap between awe (a prominent inspiring example is the infrared rainbow [7]) and understanding of NIR photography by comparing NIR and VIS photos and by discussing the physics of NIR radiation interacting with matter. 
The NIR spectral region is interesting for physics teaching because it extends the visualization of physical phenomena beyond the VIS spectral range. Some aspects of near infrared radiation have already been applied to physics education, as can be seen in a number of publications (see refs. in [4]). They deal with digital cameras, cell phones, camcorders, and webcams, all of which use NIR-sensitive silicon-based detectors.

The majority of cameras only passively detect NIR radiation, but some use an active scheme, often illuminating a scene with IR light emitting diodes (LEDs) and simultaneously detecting scattered NIR light. Passive detection usually uses the sun as radiation source. Its spectral irradiance decreases only slightly with increasing wavelength beyond the VIS range, leaving ample radiation available for NIR imaging (e.g., fig. 1 from [4]).

We specifically note that here we do not discuss other important - though non-imaging - NIR applications such as transmission of optical fibers for communication systems.

\section{2) BASICS OF NIR IMAGING}

Early NIR photography created images from photochemical reactions within special IR-sensitive films that could operate up to wavelengths of about $850 \mathrm{~nm}$. When combined with filters blocking the visible part of the spectrum, it was possible to record images using radiation from a narrow spectral range of around 750 to $850 \mathrm{~nm}$. Nowadays, modern digital cameras have focal plane array image sensors (of either CCD or CMOS type) of typically around 10 megapixels or more, which can provide much better and more conveniently acquired images.

The left line graph in Fig. 1a depicts the sensitivity of typical modern Si diodes in Amperes per Watt of incident radiation. The cut-off wavelength around $1100 \mathrm{~nm}$ is caused by the band gap in silicon of around $1.1 \mathrm{eV}$. The CCD sensor efficiencies may slightly differ from the simple Si photodiodes since the CCD arrays have additional layers and gate electrodes that can selectively absorb radiation. Also, the sensitivity depends on whether the light enters from the front or back side. Obviously, Si-based sensors are potentially able to detect near IR radiation, as indicated by the gray NIR box between 800 and 1100 nm. However, since the NIR response introduces unwanted coloration effects, camera manufacturers usually include an IR blocking filter in the camera with transmission curves similar to the ones in Fig. 2a. For NIR photography, one must remove this filter and then insert other filters that can block all or part of the visible spectrum (e.g. Fig. 2b). Whether the modified camera's auto exposure (AE) or auto focus (AF) systems work in the NIR depends on its design. (A word of caution: removing the IR-blocking filter means opening the camera case; i.e., any warranty is lost, so only a specialist should perform the necessary camera modifications).

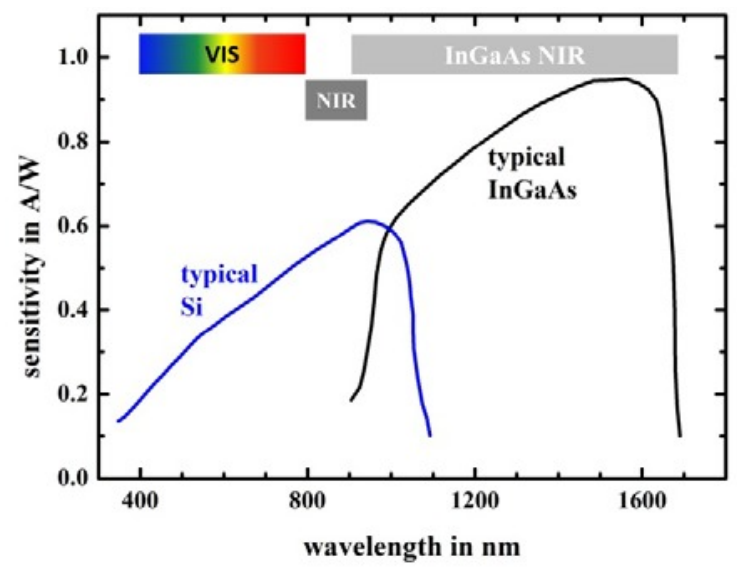

Figure 1: Typical quantum efficiency spectra of Si and InGaAs detectors (after [8]). The colored and gray bars indicate the VIS, NIR, and InGaAs NIR ranges used for detection in this work. 
The development of thermal imaging systems also led to the development of NIR cameras operating in the extended NIR range from 0.9 to $1.7 \mu \mathrm{m}$. These more expensive, quantitatively measuring cameras have focal plane array image sensors made of InGaAs, typically with fewer than 1 megapixels. The right-hand line graph in Fig. 1 also shows a typical spectral response curve of such a detector. Cameras using these detectors do not need any additional filters to block the VIS.
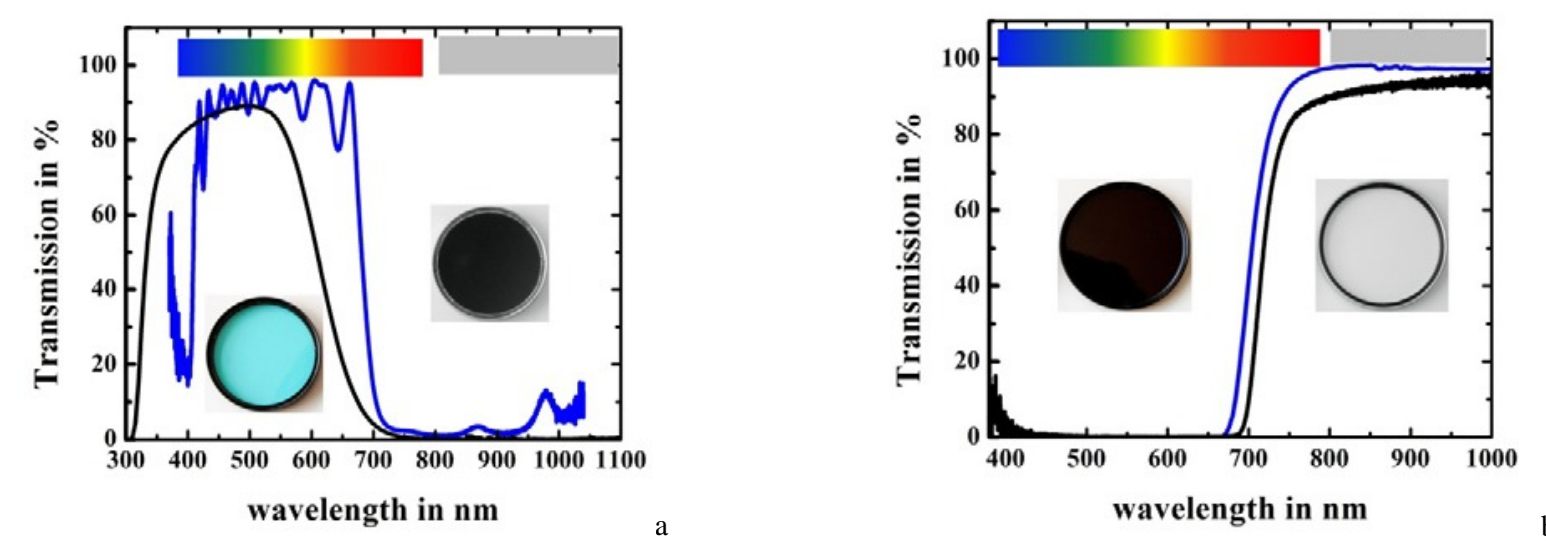

Fig. 2a) Example of two transmission spectra for typical IR-blocking filters used in digital cameras

b) Typical transmission of NIR transmission filters, i.e. VIS blocking filters. The cut-on wavelength can be shifted more or less arbitrarily. The two inserts on each graph show photos of the respective filters on top of a white sheet of paper each recorded within the VIS (left) and NIR (right) spectral range.

In the following, we show sets of images of a variety of different objects observed in the VIS spectral range with a standard camera, as well as photos detecting VIS+NIR radiation and photos detecting only NIR radiation. Photos recorded with modified DSLR cameras will be denoted as NIR photos, whereas those from the InGaAs camera will be denoted as InGaAs photos.

For each object, we discuss the respective differences in appearance arising from the underlying physics, in particular the differences in reflection and transmission spectra. First, we discuss two major differences encountered when dealing with NIR photos of regular digital cameras. Owing to the strong decrease in sensitivity towards longer wavelengths (Fig. 1), it is obvious that a much smaller signal will be detected by the camera sensors compared to VIS image recording. There is no general rule for exposure time, which can vary quite widely, depending on the camera and lens used, and, therefore, this must be tested for any given conditions. NIR exposure time will be longer than a VIS exposure time and usually a tripod is recommended. Second, if a VIS-blocking filter, such as the one shown in Fig. 2b, is used, some tiny fraction of residual VIS radiation may enter the camera and produce a false colour image whose hue will depend on the chosen white balance. Such false colour IR photos may be fascinating, but for a physics discussion it will often be easier to convert them into traditional grey scale IR photos using any common image editing program.

\section{3) SELECTED EXAMPLES}

\section{1) Vegetation and Wood effect}

In the description of the first recording of NIR photos of natural scenes in 1910, Wood already noted that NIR photos are quite different in appearance from ordinary photographs, in particular due to the extreme brilliancy of the trees and grass. This often gives the impression of seeing snow-covered landscapes. In honor of Wood's pioneering work on NIR photography, the effect of green vegetation appearing extremely bright is called the Wood effect [9]. Fig. 3 compares visible (a) with NIR (b) photos of a scene containing houses, sky, clouds, and trees with green leaves. The most obvious difference between the two photos is indeed much brighter vegetation. 
Although Wood described the phenomenon of bright NIR radiation scattering by vegetation, he did not explain it. This was done later by others. Most probably, light enters the epidermis of leaves and is scattered in deeper sections where there are many cellular walls of interior cells separated by air inclusions. This model is strongly supported by ray tracing models [10]. Multiple scattering events finally give rise to very strong backscattering, similar to VIS light back-scattering from snowcovered ground, where multiple scattering also leads to effective backscattering that yields very bright natural scenes.

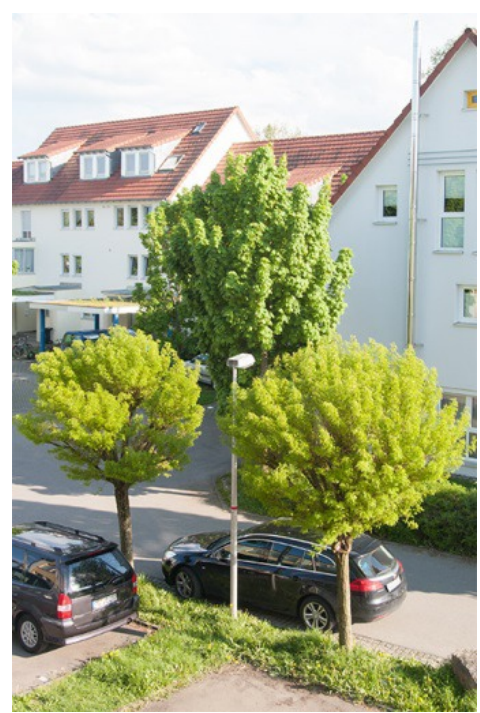

a

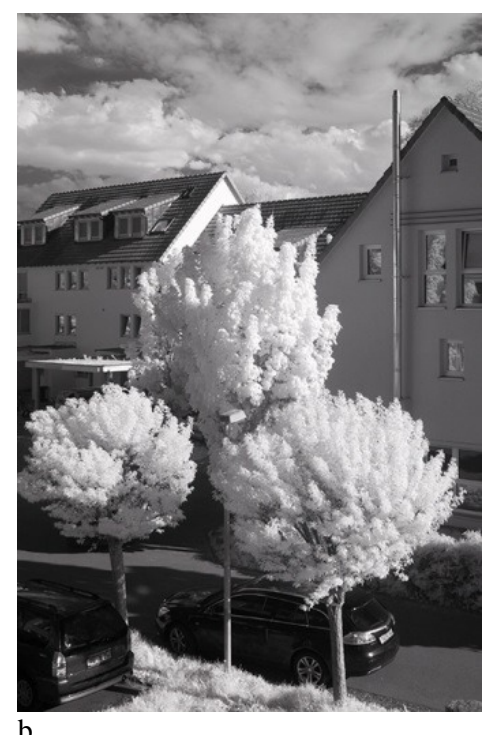

b

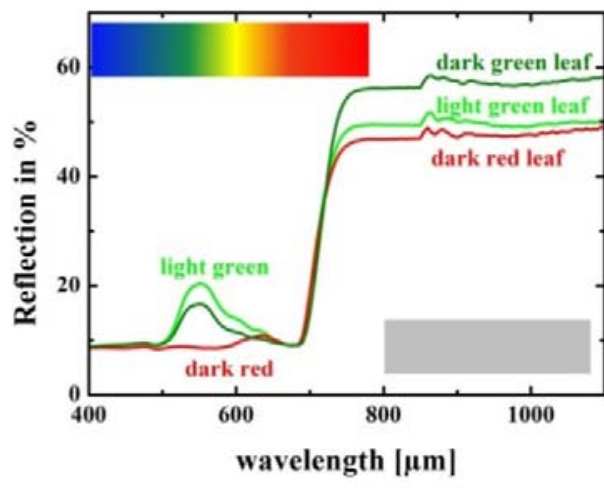

C

Fig. 3: Urban scene in VIS (a) and NIR (b) photos showing the bright scattering effect of vegetation in the NIR (Photos K. Mangold). In addition enhanced sky contrast and also the differences of reflection in the windows of the right-hand-side house are obvious. c)

Reflection spectra of two green and one red leaf measured with an integrating sphere detector that gathered light from the complete backward hemisphere. The minimum thickness of the leaves was around $100 \mu \mathrm{m}$ for the green and $200 \mu \mathrm{m}$ for the red leaf.

It is quite easy to verify the strong NIR backscattering in leaves by recording transmission and reflection spectra of sunlight through or from green vegetation. Fig. 3c shows some examples of reflection spectra $(R)$ for light and dark green, as well as dark red, leaves. These spectra were recorded with a UV-VIS-NIR Lambda 900 spectrometer (Perkin Elmer).

It is obvious that the absorption features of chlorophyll, which are responsible for photosynthesis, are only important in the VIS spectral range. At NIR wavelengths, there is much less absorption and the high reflectivity is due to diffuse scattering of radiation from index of refraction inhomogeneities within the cells, such as interfaces due to cell membranes, water accumulation, etc. For leaves of 100 to $200 \mu \mathrm{m}$ thickness, transmission can still be of the order of $50 \%$ in the NIR range and a similar amount is scattered backwards. Interestingly, the NIR behaviour appears to be independent of the visual colour of the leaf (Fig. 3b).

Because the difference of vegetation reflectance at VIS and NIR wavelengths depends strongly on chlorophyll content and plant health, it is readily possible to use NIR images to distinguish between healthy and unhealthy vegetation - even before such differences become apparent by eye (more details and refs. see [4]).

\section{2) Colored Liquids}

Many liquids that have very dark colors in the visible spectral range can appear colorless and highly transparent in the NIR. Fig.4 depicts an example of a bottle and a glass filled with a red wine. Three different images were recorded, one with normal IR cut-off filter (a: for $\lambda=400$ to $700 \mathrm{~nm}$ ), another with an IR-transmitting filter (c: for $\lambda=830 \mathrm{~nm}$ to $1100 \mathrm{~nm}$ ), and one recorded with the whole spectral range from 400 to $1100 \mathrm{~nm}$ (b). The VIS image looks familiar, showing a deep red 
color for the wine. In contrast, the NIR image makes the impression of a photo of water in a glass and clear bottle recorded in black-and-white mode, i.e. both the red wine and the green glass have turned completely transparent in the NIR range.
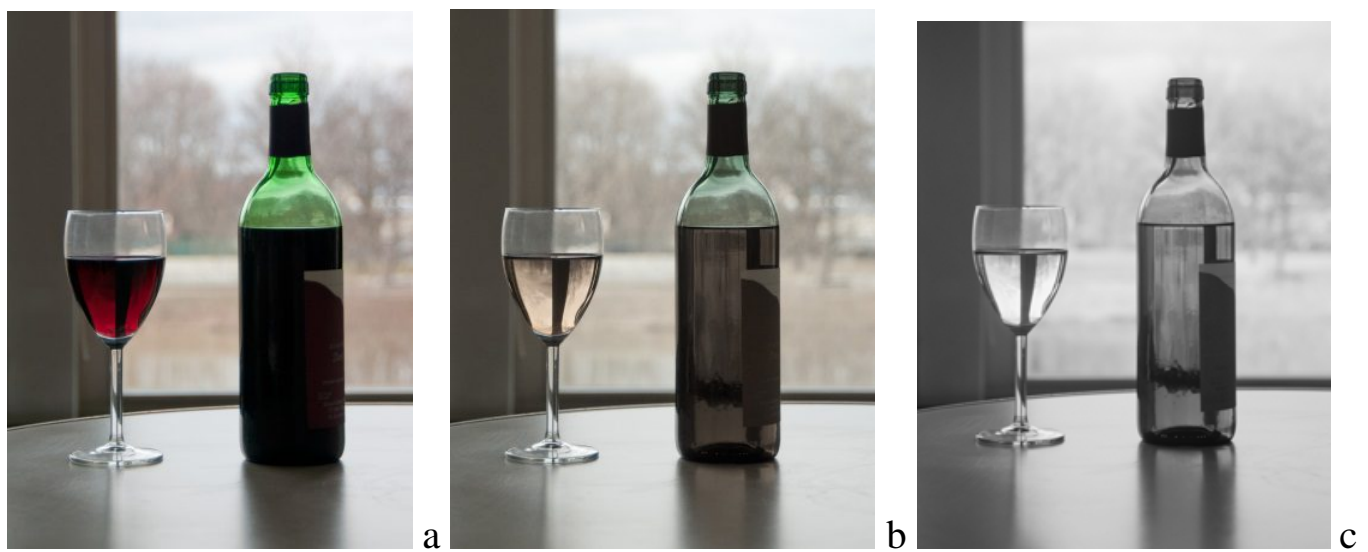

Fig. 4: Images of a bottle and glass filled with red wine recorded for VIS light only (a), whole range VIS and NIR (b) and IR only (c). Photos K. Mangold.

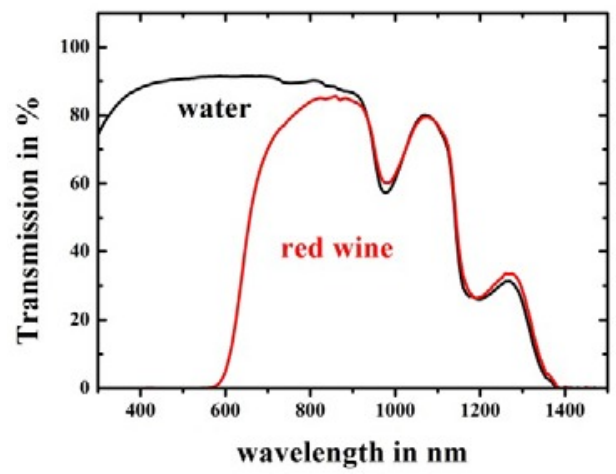

Fig. 5: Transmission spectra of red wine and water for thickness of $1 \mathrm{~cm}$.
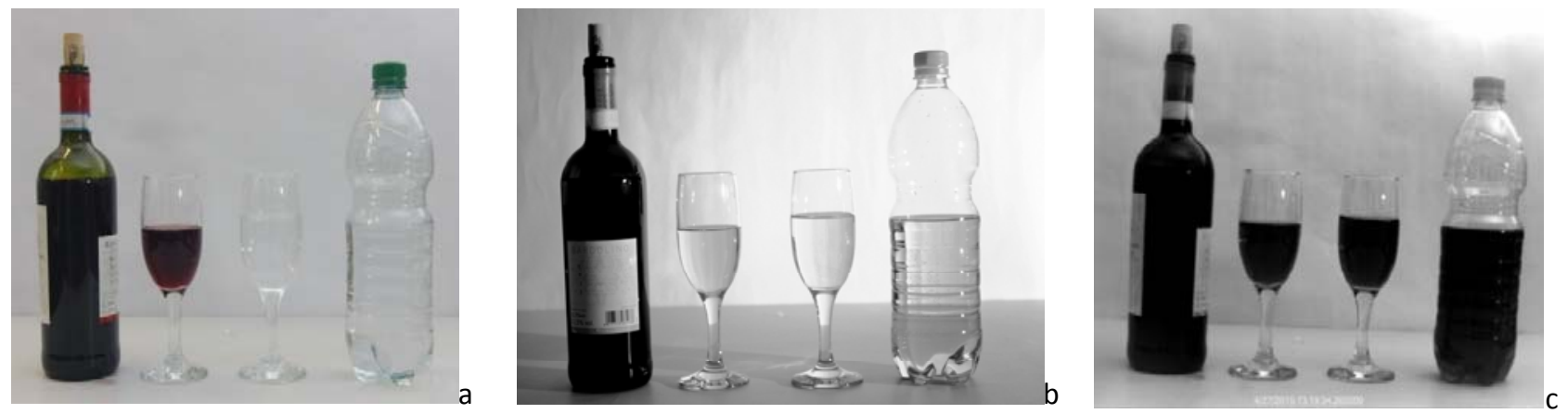

Fig. 6: Scene similar to Fig. 4 with two bottles and glasses filled with red wine (left) and water (right). a) VIS photo, (b) NIR photo recorded with Canon EOS1000 for NIR filter at cut-on wavelength $830 \mathrm{~nm}$ and (c) InGaAs photo.. 
Detecting VIS plus NIR radiation simultaneously leads to a mixture of both: the green bottle still looks a bit green and the wine in the glass still has a red tint, though not very pronounced; i.e., the IR contribution dominates the appearance in the photo. This is an excellent example of why IR cut-off filters are used in digital photography: any observer of the middle photo would be extremely puzzled and at best expect to have a wine mixed with water. Similar images can also be recorded for other liquids, e.g. diet Coke or coffee.

The explanation of this change from nearly opaque to transparent liquids is simple when studying transmission spectra of water compared to red wine (Fig. 5). The spectra were recorded for liquids in $1 \mathrm{~cm}$ glass cuvettes, hence the maximum transmission amounts to about $91 \%$ because of reflection losses. Red wine shows strong absorption features in the VIS range, with a steep increase of transmission for longer wavelengths. Similar to water, it is transparent in the NIR up to about 1400 $\mathrm{nm}$. Thicker liquid samples, such as $5 \mathrm{~cm}$ for a glass or bottle, of course show appreciably lower transmission.

Fig. 6 depicts a similar scene as in Fig.4. Two glasses are partly filled from bottles of red wine (left) and water (right). These photos were recorded with a VIS camera for a $\lambda$-range of 400 to $800 \mathrm{~nm}$ (a), a NIR camera in the range of 830 to $1100 \mathrm{~nm}$ (b), and the InGaAs camera (c) from 900 to $1700 \mathrm{~nm}$. The appearance of the liquids differs appreciably from one photo to the next. In the VIS, we have the expected behavior of transparent water but nearly opaque (i.e. very dark) wine. In the NIR, similar to Fig. 4 and explained by the spectra of Fig. 5, both water and wine look similar and are transparent. For the red wine this becomes more obvious if looking through $7.5 \mathrm{~cm}$ wine in a rectangular container (Fig. 7). The VIS image does not allow an observer to read the text behind the liquid, whereas this poses no problem for the NIR camera. Coming back to Fig.6c, the InGaAs image - if compared to the NIR image of Fig. 6b -shows both liquids as nearly opaque, which is surprising at first glance.
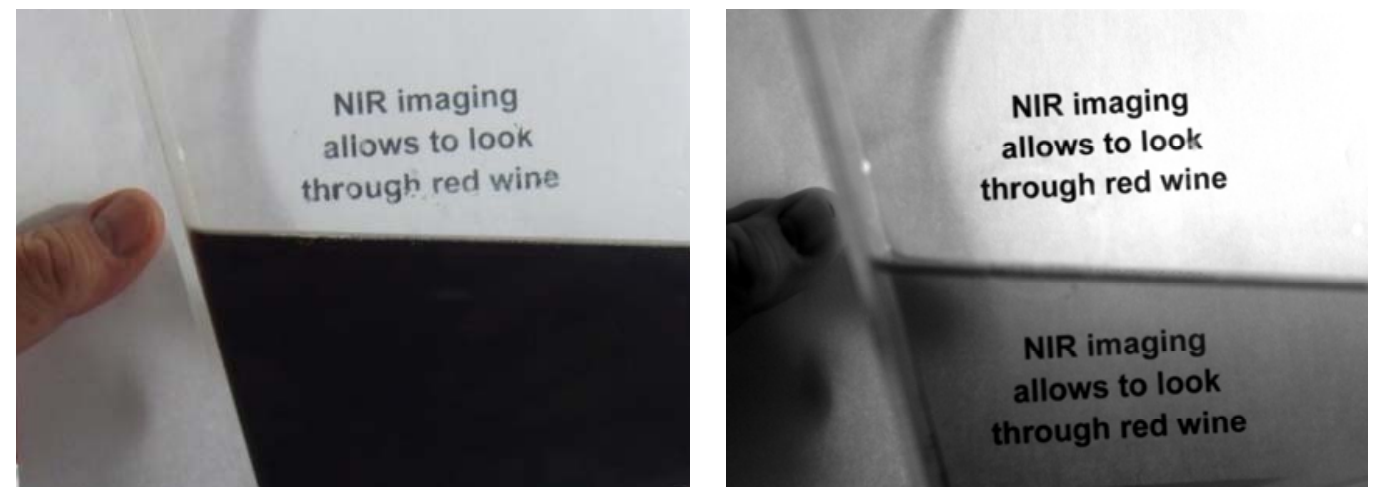

Fig. 7: VIS (a) and NIR photo (b) of text observed through $7.5 \mathrm{~cm}$ of red wine.

According to the spectra of Fig.5 and the detector sensitivities (Fig.1), one may have expected a transmission similar to that of the NIR camera. The explanation is simple. The signal is due to the detector sensitivity, which for InGaAs is still rising for the small wavelengths where the $5 \mathrm{~cm}$ thick wine samples still have some transmission. As a consequence, the detectorwaveband-integrated transmission signal of wine and water only amounts to a few percent of the transmission of a nonabsorbing specimen; i.e., it must appear very dark.

\section{3) Looking through paint}

There are some objects, such as paints and varnishes, which are visually opaque but yet show high transmission in the NIR. This feature has already been used as a physics trick in demonstration experiments [11]. Fig. 8 depicts spectra of standard transparencies used for demonstrations on overhead projectors and others covered with black overhead markers. The ink from overhead transparency markers makes it impossible to look through a transparency or - if marked on paper - to see the underlying text in the visible spectral range (Fig. 9a). In contrast, this is possible in the NIR, due to its transmission above $800 \mathrm{~nm}$ (Fig. 9b,c). These examples can be demonstrated nicely in the classroom. 


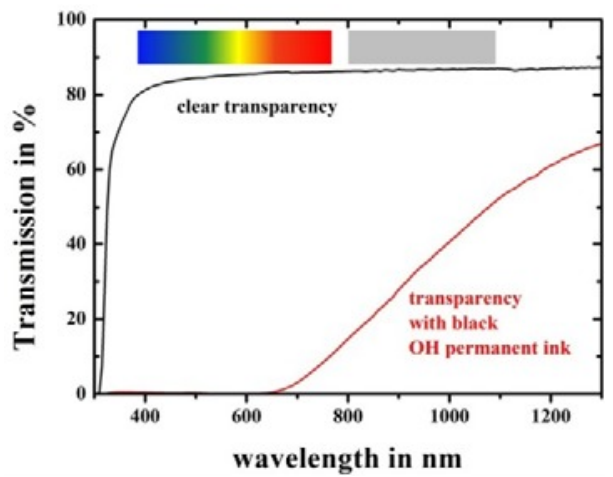

Fig. 8: Spectra of clear transparency and the same transparency partly covered with the same ink.
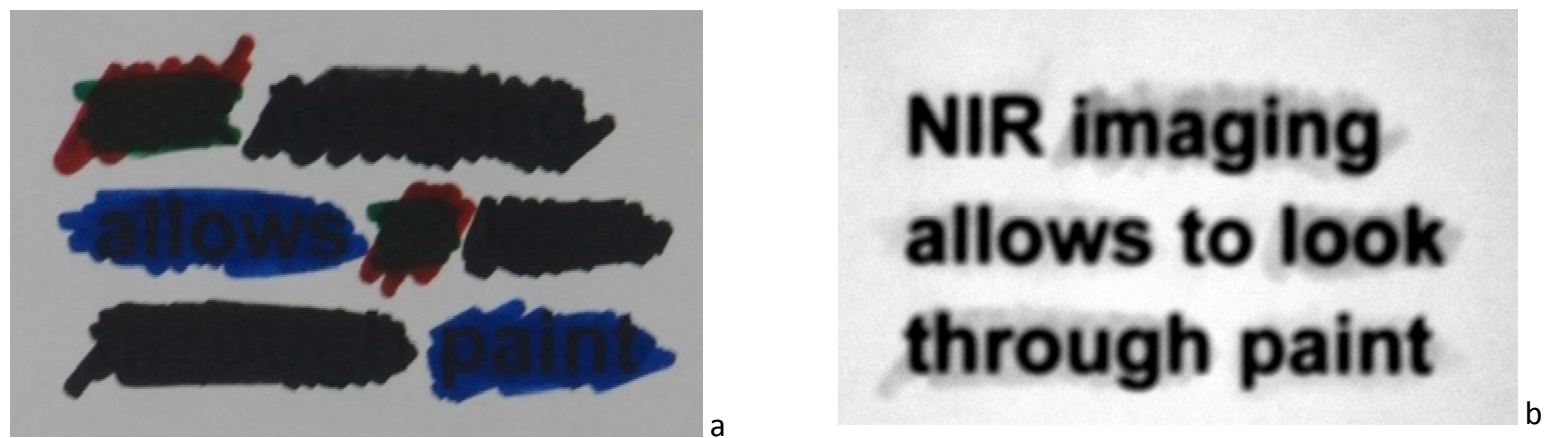

\section{NIR imaging allows to look through paint}

Fig. 9 VIS (a), NIR (b) and InGaAs (v) photo of black text from a laser printer on regular paper observed through various colored text markers. In the VIS, one may just have a chance to see through the blue color. In contrast the NIR, and even better, the InGaAs photo allows an observer to read the text very clearly.

\section{4)}

More examples

Besides the Wood effect, Fig. 3 also showed partly cloudy and hazy skies. Apparently, there is a much higher contrast between the clear sky and the clouds for the NIR image. This is due to the scattering characteristics of molecules and the much larger haze or cloud droplets. Molecular scattering by atmospheric gases follows Rayleigh's law, i.e. a rapid $(1 / \lambda)^{4}$ decrease with wavelength. In contrast, much larger cloud droplets scatter radiation according to Mie scattering, which has a less pronounced wavelength dependence. The consequence of higher NIR contrast between dark clear sky and brighter clouds was discussed more quantitatively from spectra of scattered sky light and diffusely scattered cloud light (see [4]). 
Other spectacular effects of NIR photography become apparent when analyzing photos of people. The skin behaves quite different in the VIS and NIR ranges (see Figs. in ref [4,5]). Human tissue is a highly inhomogeneous material containing chromophores, blood vessels, and light-scattering particles and fibres whose sizes differ appreciably. Therefore the optical properties of biological tissue in general, and human skin in particular, must take into account a large amount of multiple scattering events within the various layers of the skin. As a result, the 1/e penetration depth of optical radiation can become much larger for NIR radiation than for VIS light. For example, penetration depth values can range between $90 \mu \mathrm{m}$ for blue light $(\lambda=400 \mathrm{~nm})$ and $1.6 \mathrm{~mm}$ for NIR radiation $(\lambda=1000 \mathrm{~nm})$. As a consequence, veins may be visualized even if they are a few mm below the surface [12]. Image contrast is due to a combination of NIR scattering in the tissue and absorption by hemoglobin in the blood vessels. This phenomenon has been applied [12] to enhance localization of subsurface blood vessels needed for blood withdrawal from small children. The blood vessels were usually located at depths from 1 to several mm and the method worked successfully in hands or wrists and also at the inside of the elbow of children. High-power NIR LED's around $\lambda=850 \mathrm{~nm}$ were used underneath the puncture sites and images were captured from above.

\section{4) CONCLUSIONS}

Modern digital cameras combined with simple optical filters provide excellent tools for observing the near-infrared world. They allow us to teach how simple physics principles can be used to explain the unusual appearance of some everyday objects and scenes in the NIR. We have presented example images recorded using visible and near-infrared radiation, and have shown how measured reflection and transmission spectra can be used to explain the resulting different appearances.

\section{5) REFERENCES}

[1] Möllmann, K.-P. and Vollmer, M., “Infrared Thermal Imaging: Fundamentals, Research and Applications,” Wiley (2010).

[2] Möllmann, K.-P. and Vollmer, M., “Infrared thermal imaging as a tool in university physics education,” Eur. J. Phys. 28, S37-S50 (2007).

[3] Möllmann, K.-P. and Vollmer, M., “Teaching Fourier Transform Infrared Spectroscopy in physics lab courses,” Eur. J. Phys. 34/6, S123-37 (2013).

[4] Mangold, K, Shaw, J.A., and Vollmer, M., “The Physics of Near-Infrared Photography,” Eur. J. Phys. 34/6, S51-71 (2013).

[5] Mangold, K, Shaw, J.A., and Vollmer, M., “Rotwein zu Wasser,” Physik in unserer Zeit 46/1, 12-16 (2015).

[6] Sandidge, D., "Digital Infrared Photography,” Wiley (2009).

[7] Greenler, R.G., “Infrared Rainbow, ” Science vol. 24, no. 4003, pp. 1231-1232 (1971).

[8] Hamamatsu InGaAs selection guide, March 2015, https://www.hamamatsu.com/resources/pdf/ssd/ingaas_kird0005e.pdf

[9] Finney, A., “Infrared photography,” pp. 556-562 in Peres, M.R. (ed.), Focal encyclopedia of photography, 4th edition, Elsevier, Amsterdam (2007).

[10] Allen, W.A., Gausman, H. W., Richardson, A. J. , "Willstatter-Stoll Theory of Leaf Reflectance Evaluated by Ray Tracing,” App. Opt. 12/10, 2448-2453 (1973).

[11] Micklavzina, S. , “Tricks with invisible light,” Physics Education 38, 492-494 (2003).

[12] Cupera, N.J. et al, "The use of near-infrared light for safe and effective visualization of subsurface blood vessels to facilitate blood withdrawal in children,” Medical Engineering \& Physics 35 /4, 433-440 (2013). 